\title{
Superviviencia en cáncer de laringe: A propósito de un artículo
}

Felipe Cardemil M ${ }^{1}$.

Recientemente, Orellana y cols han publicado un artículo analizando resultados oncológicos de una serie clínica de pacientes con cáncer de laringe ${ }^{1}$. Sin embargo, a la luz de lo publicado, es necesario plantear algunas inquietudes.

En primer lugar, mencionan haber encontrado 52 casos con diagnóstico de cáncer en un período de 6 años. Como ellos mismos mencionan, el Complejo Asistencial Doctor Sótero del Río (CASR) tiene una población asignada de 1.521.144 habitantes. Asumiendo que 70\% de la población del Servicio de Salud Metropolitano Sur-Oriente se atienden en la red pública de salud, y asumiendo una incidencia ajustada muy conservadora a la baja de 1,8 casos nuevos de cáncer laríngeo en hombres por cada 100.000 habitantes $^{2}$, se esperarían encontrar alrededor de 27 casos nuevos por año, lo que representaría alrededor de 160 casos durante el período de estudio. Si $70 \%$ de estos casos recibió tratamiento en la red pública de salud, deberían ser alrededor de 113 casos confirmados de cáncer laríngeo tratados en el CASR durante ese período. La menor cantidad de casos encontrados para el estudio plantea diferentes preguntas respecto a qué sucedió con ellos. Este aspecto tiene una influencia en los resultados del estudio, y pone acento respecto de si el tipo de metodología utilizada para encontrar los casos fue el más apropiado para este análisis o no.

En segundo lugar, los autores mencionan que 22 casos (58\%) de la cohorte se presentaron al diagnóstico con un cáncer T4a, y que 17 casos fueron manejados con laringectomía total (con o sin coadyuvancia) (Tabla 4). Sin embargo, en la Tabla 3 se menciona el manejo de 7 casos en estadio precoz, y en la Tabla 4 se reporta la distribución del tratamiento de 27 casos de estadio avanzado, dando un total de 34 casos. No se hace mención a los restantes 4 casos, del total de 38 casos incluidos finalmente en el estudio. Además, existe relativo consenso en la actualidad, incluso dentro de líderes en el campo de la radioterapia ${ }^{3}$, que se debe ser muy cuidadoso al recomendar terapia de órgano preservación no quirúrgica como manejo de la enfermedad laríngea T4a, ya que no existe evidencia nivel I que apoye estrategias de órgano preservación no quirúrgicas en estos $\operatorname{casos}^{3}$, debido a los criterios de exclusión del RTOG 91-11. Específicamente en enfermedad T4a, la cirugía primaria sumado a coadyuvancia se asocia a mayores tasas de control local.

Por último, los autores reportan una superviviencia a 5 años de $42 \%$ para cáncer avanzado de laringe (etapas III y IV). De acuerdo a lo que se deduce del artículo, éstos son datos de supervivencia global (overall survival). Sin embargo, las cifras reportadas en el artículo que ellos citan son de supervivencia relativa (net survival $)^{4}$, que representa una medida de supervivencia de cáncer en ausencia de otras causas de muerte, calculada como la razón entre la proporción de supervivientes de una cohorte de pacientes con cáncer sobre la proporción de supervivientes esperados en un grupo de personas sanas que sea comparable. En este sentido, una revisión sis-

1 Departamento de Otorrinolaringología y Departamento de Oncología Básico Clínica, Facultad de Medicina, Universidad de Chile. felipecardemil@med.uchile.cl 
temática de la literatura reportó que la supervivencia global en enfermedad laríngea T4a (que corresponde a etapa IV, que tiene una supervivencia inferior a etapa III) a 5 años varío entre $16 \%$ y $50,4 \%$ para pacientes tratados con órgano preservación con quimiorradioterapia y $10 \%$ a $80,9 \%$ con cirugía con coadyuvancia 5 . Más aún, recientemente una publicación norteamericana analizó los resultados de una estrategia con neoadyuvancia y selección individualizada en base a la respuesta a ésta, reportando una supervivencia global en cáncer laríngeo avanzado, independiente de la modalidad terapéutica, de $72 \%$ a 5 años ${ }^{6}$.

Orellana y cols han publicado un artículo importante: es necesario analizar y someter a discusión los resultados oncológicos en oncología de cabeza y cuello en Chile. Sin embargo, algunos planteamientos del artículo podrían ser sometidos a discusión, desde el punto de vista del funcionamiento de la red de salud (¿qué sucede con los casos que no están llegando al hospital?), de los registros hospitalarios de cáncer (cada vez más necesarios), y de los resultados propiamente tales. En oncología, el análisis de supervivencia es el desenlace más relevante, y es fundamental que todos los centros que manejan pacientes con cáncer realicen auditorías de resultados. Es la única manera en la que el sistema puede buscar soluciones e intentar optimizar los resultados.

Felipe Cardemil M Departamento de Otorrinolaringología y Departamento de Oncología Básico Clínica, Facultad de Medicina, Universidad de Chile. felipecardemil@med.uchile.cl

\section{REFERENCIAS}

1. Orellana MJ, Chuang A, Fulle A, Fernandez R, Loyola F, Imarai C. Cáncer de laringe: Serie de casos en 6 años en el Complejo Asistencial Doctor Sótero del Río. Rev. Otorrinolaringol. Cir. Cabeza Cuello 2017; 77: 35-43.

2. Ministerio de Salud, Chile. Primer Informe De Registros Poblacionales De Cáncer De Chile, Quinquenio, Incidencia, Mortalidad y Estimación País (2003-2007). 2012. Disponible en http://maquetas.ciiet.cl/elgg/file/download/5596 (revisado 1 de Mayo del 2017).

3. Forastiere AA, Weber RS, Trotti A. Organ Preservation for Advanced Larynx Cancer: Issues and Outcomes. J Clin Oncol 2015; 33(29): 3262-8.

4. American Cancer Society. Laryngeal and hypopahryngeal cancers overview: Survival rates for laryngeal and hypopharygeal cancers by stage. Disponible en: http:www.cancer.org/cancer/laryngealandhypopharyngealcancer/ detailedguide/ laryngeal-and-hipopharyngeal- cancer-survival-rates (revisado 1 de Mayo del 2017).

5. Francis E, Matar N, Khoueir N, Nassif C, Farah C, Haddad A. T4a laryngeal cancer survival: Retrospective institutional analysis and systematic review. Laryngoscope 2014; 124: 1618-23.

6. Wolf Gt, Bellile E, Eisbruch A, Urba S, Bradford CR, Peterson L. Survival Rates Using Individualized Bioselection Treatment Methods in Patients With Advanced Laryngeal Cancer. JAMA Otolaryngol Head Neck Surg 2017; 143(4): 355-66. 